SOME PRINCIPLES OF PLASTIC SURGERY : INCISIONS, CONTOUR, AND THE SUTURE.

By J. L. AYMARD, M.R.C.S., L.R.C.P. LoND., TEMPORARY CAPTAN, R.A.M.C., QUEEN'S hospitat, FrogNat, SIDCUP, KENT.

THE prime factors of artistic work in the department of plastic surgery are the restoration of contour and the correct method of suturing. The disappointing appearance of much careful plastic work is vaguely recognised by the untrained observer who has any artistic perception; the trained artist sees at once that the essential thing which is so frequently absent from work which is otherwise admirable is the right degree of fullness which would have restored a flat surface to its normal contour.

The three important factors, which I propose to deal with here, necessary to bring about a contour as nearly normal as possible are: first, the correct method of incising the skin ; secondly, the manipulation of the deeper structures; and, thirdly, the best method of bringing about satisfactory union. The other equally important factors, such as freedom from sepsis, condition of patient, \&c, , are already well known.

The Skin Incision.

In dealing with the question of skin incision we are, as far as I know, limited to two methods : the one in which the

FIG. 1.$$
\text { A }
$$

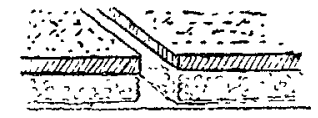

$\mathbf{B}$

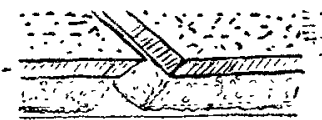

C

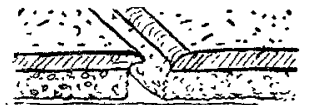

D

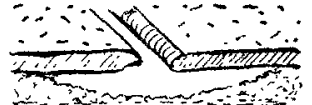

E

F

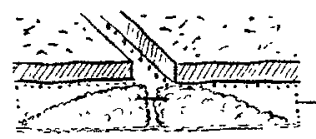

C

$\mathrm{H}$
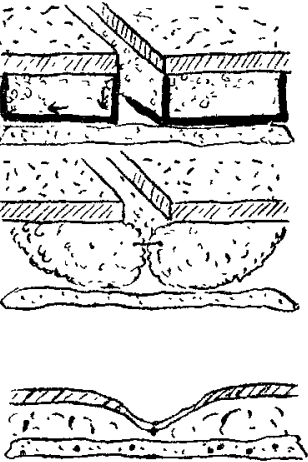

Orinary incision at right angles to surface of skin. B, Author's incision upon slant.

c. Appearance assumed imm diately after incision.

$\mathrm{D}$, To demonstrate undercutting of skin.

E, Ordinary fat flap.

$F$, Author's deep-tissue flap

incision for.

, Author's deep-tissue flap

rolled in position.

H. - Deep scar with loss of tissue. skin is divided at right angles to the surface and the other upon the slant. The latter method was fully described in Тнस LANCET some 11 years ago ${ }^{1}$ and demonstrated by me in Sir Arbuthnot Lane's clinic at Guy's Hospital. Two years later it was discovered by the Germans and later by the Americans, in each case without acknowledgment. Having practised this incision continuously since this period, I may be permitted to draw attention to its value again, especially since it is now so frequently used in plastic surgery in this country to-day.

A, Fig. 1, is intended to represent the usual incision at right angles to the surface; $B$, that in which the skin is incised upon the slant. At the moment of incision the actual appearance is doubtless as represented, but almost immediately the condition of $\mathrm{C}$ is produced. The upper flap assumes a curved form and the under one a convex. The actual edge of the skin of the upper flappresents an appearance (not illustrated) resembling the cutting edge of a saw and not unlike a fringe. This condition is due to the fact that the skin is not flat, but, on the contrary, a series of ridges and holes. This ragged edge must often have been observed by surgeons when commencing the incision for a Thiersch graft. The surgical importance of the contracted rolled-in edge of the upper flap is that it is liable to be cut off if the knife is introduced twice instead of making one clean incision. My slanting incision is very suitable for incisions in the neighbourhood of the cheek, the mouth, the chin, and the forehead, but around the eye and in the neck, where the skin is loose and mobile, the right-angle one is the more suitable.

1 Invisible Surgery, by J. L. Aymard, THx LıNcer, May 12th, 1906.
Method of Dealing with the Deeper Structures.

Having dealt with the skin incisions, we have next to con sider how the deeper tissues must be dealt with in order to fill up the gap produced by the excision of a depressed scar as illustrated at $H$, Fig. 1 . Leaving out the question of fres grafts and the introduction of foreign bodies, we have only the fat and neighbouring tissues to make use of. Provided the depression is only slight, simple undercutting, as represented at $D$, may suffice. A common method, however, is to undercut the skin and divide it from the fat, as represented by the dotted line in $\mathrm{F}$, rolling it together as shown, but the method I much prefer and now almost always employ is shown in F. The thick black lines represent the undercutting straight down to the bone, and in the direction of the arrow marks up to the skin; the block is thus dependent upon the skin flap for its bloodsupply. These blocks of

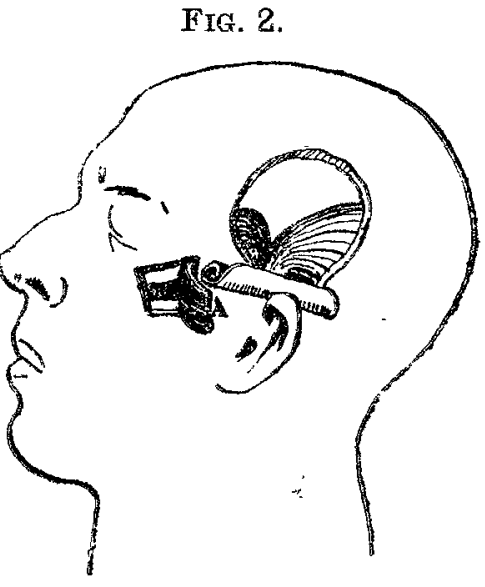

Major H, D. Gillies's temporal muscle flap. tissue can then be easily rolled inward, as $G$, and secured with catgut, thus filling up the gap and restoring the contour.

An ingenious method of deep-tissue adjustment is that devised by my colleague, Major H. D. Gillies, to fill up depressions caused by loss of malar bones. The diagram, Fig. 2, shows how he partially separates, and turns down under a bridge of skin, a portion of the temporal muscle. I have seen some excellent results due to the employment of this method.

\section{Method of Suturing.}

The next question is how to adjust the skin. Until fairly recently I have endeavoured to bring the edges of my incision into perfect apposition with the hope that they would so remain, but, in a large plastic operation, with the knowledge that I should have to recognise that the result was ordinarily successful if as many as twothirds of my sutures held in position, taking into consideration all the troubles so frequently attending such an extensive operation, I am now satisfied to sacrifice any immediate cosmetic appearance in the theatre for what I know to be the better method of attaining an ultimately good result. I have long suspected that the appearance of $a$ wound two days after operation was deceptive-that is to say, that whilst the interrupted suture still held, the cuticle only was in contact, and that a condition resulting in $\mathrm{D}, \mathrm{Fjg}$. 3 , was, in fact, in existence, to be followed by $\mathrm{E}$, or in the long run the alternative of an otherwise unaccountably wide scar.

Grery general surgeon must have been struck by the small linear scar invariably produced by the use of the Michel clip, A, Fig. 3, which is clearly A, Michel's clipas ordinarily used. due to the apposition of a sively used by author. substantial surface of the $c$, Interrupted suture. two edges which subsequently $D$, Appearance of skin edge with

E, Complete break-down, appearthe normal position. Whether ance of.

the skin be cut upon the slant or at right angles I now almost entirely employ the mattress suture (B, Fig. 3), which gives the same result as the Michel clip. The clip is not suitable for the face, because it occupies too much space and is difficult and painful to remove in this region. The mattress horsehair suture, on the contrary, cuts quickly through the 
cuticle, but holds the deeper tissues until union is secured, despite moderate tension and even suppuration, should it occur. This, however, is not meant to imply that I neglect in the least the additional safeguard of that most valuable adjunct in plastic surgery-the relaxation suture, whether in the form of the corrugated vulcanite disc with silkworm gut or my wire and clip methud.

\section{A METHOD OF APPLYING ANTISEPTICS TO THE DEEPER PARTS OF WOUNDS.}

\section{BY THOMAS H. KELLOCK, M.C. CAMB., F.R.C.S. ENG., CAPTAIN, R.A.M.C. (T.)}

THE multitude of septic wounds at the present time, which occupy the attention of so many medical men, has very naturally given rise to the use of a variety of antiseptics and many methods of application. To judge of the efficiency of the former due attention should be paid to the latter, and it is obviously unfair to judge of the value of an antiseptic when it is only applied to the part of a wound that is visible. In every wound of any magnitude there must be recesses and deep parts into which, however well such a wound may be drained, the antiseptic applied in this way never reaches and where organisms may collect and multiply unmolested.

The Carrel method of irrigating wounds has done a good deal to improve their treatment and has drawn attention to the advantage of applying antiseptics to the deeper as well as to the more superficial parts of wounds. The method, however, has certain disadvantages. The tubes generally used are perforated with round holes for some distance from the closed end, and if there be much resistance to the exit of fluid at the deepest part the solution finds its way through the openings nearer the surface of the wound and so the deeper parts are not reached.

These tubes, too, act to a certain extent as drains. Pus finds its way into them through the openings and is washed back into the wound again when fluid is allowed to flow. Another disadvantage of the method is the necessity of keeping the patient still. Movement on his part is apt to cause the tubes to be drawn out of the wounds, and it is not at all an uncommon experience to find tubes that were originally inserted deeply into a wound lying only just under, or even on, the surface.

Attempting to improve on this, I have found the following method of much service. Taking a piece of drainage-tube about 8 or 10 inches in length and of calibre about equal to that of a No. 8 catheter, the end is securely closed by a ligature and a small oblique incision made with sharp scissors as near as possible to the closed end. The incision should cut a small valve with apex directed towards the closed end-the more oblique it is the better-and should at its base divide one-third of the circumference of the tabe (see figure, A). This incision will be found to act as a most
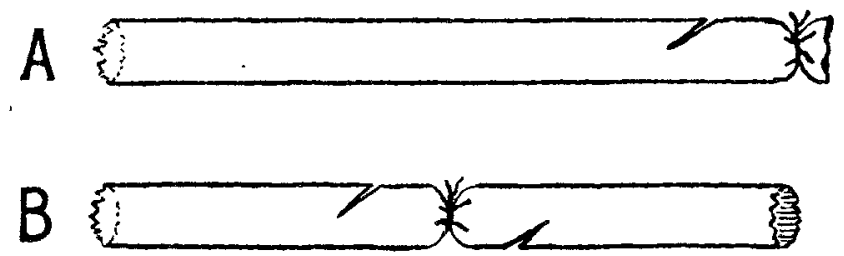

efficient valve, opening under very slight pressure from within and preventing any regurgitation of pus or of the irrigated fluid. The tied end should be inserted to the very depth of the wound and the free end of the tube left projecting through the dressings; the number of tubes used must depend on the size and character of the wound, but it will generally be found that one, or at the most two, will suffice.

In cases of a perforating wound of a limb or of a wound with two openings widely separated a very useful modification is to ligature the tube at its centre and cut a valve on each side of the ligature and pointing towards it (B). This is passed through the wound and the irrigation done from both ends. In this way any fluid that is irrigated into the free ends of the tube must certainly find its way to the very bottom of the wound and from there towards the surface.

To secure the tubes in position a useful plan is to make a small independent incision through the skin near the edge of the wound just large enough to grip the tube without occluding its lumen, and the free end of the tube is drawn through this after the tied end has been passed to the bottom of the wound. The free ends of the tubes are left outside the dressing and the irrigating fluid passed into them by means of a glass syringe. Gravity alone will generally suffice, but, if necessary, a little assistance may be given by using the plunger of the syringe. If only a small quantity of fluid be used at a time the dressings on the wound need only occasional changing. I have been using flavine for this purpose. One to two drachms of a 1 in 1000 solution is injected through the tubes twice in 24 hours, and the results in some very septic cases have been most satisfactory.

A question on which I have not yet been able to satisfy myself completely is whether wounds treated in this way should be drained. If free drainage is provided, the antiseptic fluid very readily finds its way to the surface and is absorbed by the dressings, and so is not able to remain in contact with the real surface of the wound. I have sometimes thought that the best results have come about when the drainage is not free. The walls of the wound then remain open and the mixture of pus and antiseptic fluid is brought into contact with the suppurating surface at all points.

\section{Clinital gittes:}

MEDICAL, SURGICAL, OBSTETRICAL, AND THERAPEUTICAL.

\section{MEMBRANOUS CONJUNCTIVITIS FOLLOWING A SQUINT OPERATION.}

\section{BY S. Johnson TAYLOR, M.B.Edin.}

RECENTLY I performed an advancement cum tenotomy operation for a convergent squint, in a girl aged 11 years, followed by a complication which I believe to be very rare. I used three stitches for the advancement and one for the tenotomy part, which I did, as usual, by the open method. All went well. Two days later the upper eyelid was a little more swollen and glazed than is usual and the advancementwound region did not look quite nice; this was soon succeeded by a definite dirty membranous area which I diagnosed clinically as diphtheritic, the tenotomy-wound area quite escaping. I at once had antitoxin injected hypodermically and used quinine lotion freely and frequently, as advised by Sir John Tweedy in "Diphtheritic Conjunctivitis," and slowly the condition cleared up; the stitches became very loose and either were cut out or came away. The sloughy membranous patch involving the tendon and distal end of the external rectus was loth to disappear and did so very gradually, leaving a swollen granulation patch and a convergent squint as marked as before the operation. The general systemic disturbance was very slight. No bacteriological examination was made or was necessary, the clinical condition being quite sufficient to label the disease. The patient had diphtheria last Christmas and two other of the children had it, and the baby with cleft palate has been for some weeks-before the operation-and still is in our isolation hospital as a carrier of the complaint.

The ground behind the patient's house is soaked with sewage, there being no drainage system there. I have mentioned this case to several of my London colleagues, but can find no parallel thereto. The one attack of general diphtheria did not confer any immunity against the local affection. I venture to state that this complication of a squint operation is excessively rare if not unique.

Norwich.

\section{THE CLINICAL MANIFESTATIONS OF A DOUBTFUL CASE.}

By M. J. Petty, M.B. Cayb., F.R.C.S. Eng.

THE following case is recorded as a problem in diagnosis. Patjent, girl, 6 years of age. No evidence of other cases in family. Mother and father both healthy. When patient was 2 years of age a slight fit, with loss of consciousness, occurred; apparently complete recovery. Four months later the child began to drag right foot along the ground, gince then progressively getting worse till whole of right side of body was noticed to be affected. Occasional 